\title{
Journals question integrity of almost 200 papers by Japanese anaesthetist
}

In the print version of this News article about Dr Yoshitaka Fujii of Toho University, Tokyo (BMJ 2012;344:e2490, doi:10. 1136/bmj.e2490), we inadvertently used a picture of a different Dr Yoshitaka Fujii, who is not the person mentioned in the

article. We apologise to Dr Fujii for our mistake. 